\title{
Novedades del tucán del Pacífico Rhamphastos brevis: hábitat y comportamiento en tres localidades del departamento del Chocó, Colombia
}

\author{
Newness of tucan del Pacífico Rhamphastos brevis: habitat and \\ behavior in three localities of the department of Chocó, Colombia
}

\author{
Jimi Moya R. *, Jorge V. Dunlap*, Nerlen A. Sinisterra* \\ RESUMEN
}

Es relevante resaltar que esta investigación documenta por primera vez los aspectos más importantes del comportamiento y el uso del hábitat del tucán del Pacífico. Las visitas a tres localidades del departamento del Chocó, las estaciones fijas, los recorridos y monitoreos constantes ayudados de binoculares (10x 40) y miras telescópicas, permitieron documentar el comportamiento (hábitos grupales, solitarios, parejas), actividad, hábitat, tipo de dieta y afinidad biogeográfica, mediante observaciones directas. En general, se encontró que el tucán del Pacífico es más conspicuo en zonas abiertas, en el ecotono y donde predominan cultivos de pancoger; utiliza los estratos más altos del bosque donde frecuenta algunas palmas (meme, donpedrito, milpesos) y el yarumo uva, aprovechar sus frutos y desarrollar actividades de acicalamiento y descanso.

Palabras clave: Tucán del Pacífico; Hábitat; Comportamiento; Chocó; Bosque pluvial tropical.

\section{ABSTRACT}

It is relevant to note that this study documents for the first time the most important aspects of behavior and habitat use of Pacific toucan. Visits to three sites in the department of Chocó, the fixed stations, routes and constant monitoring, aided by binoculars $(10 x 40)$ and scopes, to document the behavior (habits group, individuals, couples), activity, habitat type diet and biogeography affinity through direct observation. In general it was found that the Pacific toucan is most conspicuous in open areas in the ecotone, and where most food crops, using the highest strata of the forest where frequent some palms (meme, donpedrito, milpesos) and yarumo uva leveraging the benefits of developing them and grooming activities and rest.

Keywords: Toucan; Habitat; Behavior; Chocó; Rain tropical forest.
* Biólogo Contratista, Instituto de Investigaciones Ambientales del Pacífico (IIAP), Quibdó, Colombia. e-mail:jimhalver68@gmail.com jorgedunlap@hotmail.com nasalex7478@hotmail.com Recibido: 28 de marzo de 2011 Aceptado: 20 de abril de 2011

\section{INTRODUCCIÓN}

El tucán del Pacífico Rhamphastos brevis, es un ave perteneciente a la familia Ramphastidae; habita en bosques húmedos y a menudo frecuenta márgenes o bordes de ecosistemas selváticos. La especie ha sido registrada hasta los 1000 msnm por Hilty y Brown $(2001,2009)$, Mcmullan etal. (2010) y Restall et al. (2006), quienes la registran desde la región media del Chocó con distribución vertical inferior a los
$1200 \mathrm{msnm}$, pero se cree que puede llegar hasta los $1500 \mathrm{msnm}$. $R$. brevis es casi endémica (C-end) para la provincia chocoana y se extiende a naciones vecinas como Panamá y Ecuador. Por esta razón la especie se puede encontrar en el suroccidente de Panamá, en el occidente de Colombia y en la región noroccidental de Ecuador. En Colombia se encuentra en el norte del departamento del Chocó (Tacarcuna, PNNKatios, serranía del Baudó) y en los departamentos de Cauca, Valle del Cauca y Nariño. 
A pesar de ser un ave importante para la conservación por su estrecho rango de distribución, $R$. brevis es un ave poco estudiada y la información biológica de esta especie es limitada. Por esta razón, el estudio del comportamiento y el uso del hábitat del tucán del Pacífico, se constituyen en elementos de vital importancia en la construcción de la historia de vida de esta especie . Asimismo, provee una serie de insumos que permiten conocer las interrelaciones de la especie con su entorno natural.

La generación de conocimiento a partir de este novedoso trabajo es, además, un valioso aporte al conocimiento de la avifauna del Pacífico colombiano y un primer paso hacia la creación de herramientas y estrategias clave, para generar acciones en torno al manejo y conservación de las poblaciones del tucán del Pacífico Ramphastos brevis.

El objetivo general del presente artículo es brindar información del uso del hábitat de la especie, así como algunas conductas de comportamiento observadas en tres localidades del departamento del Chocó, como un aporte dirigido hacia un mejor entendimiento de la historia natural de la especie.

\section{ÁREADEESTUDIO}

El estudio se realizó en el departamento del Chocó y para su desarrollo se tomaron en cuenta tres zonas importantes para el monitoreo de poblaciones animales: la Estación Ambiental Tutunendo (EAT) y la Estación Ambiental Alto San Juan (EAASJ), que corresponden a áreas de reserva protegidas por el IIAP, donde se realizan actividades conjuntas de investigación y conservación con los habitantes de las comunidades locales que allí se asientan; la otra zona de trabajo corresponde a un sector del corregimiento de Opogodó (municipio de Condoto). Esta localidad se seleccionó estratégicamente por hacer parte del área de influencia directa de un proyecto de explotación petrolera desarrollado por la Agencia Nacional de Hidrocarburos (ANH), principal entidad financiadora del proyecto. Esta zona, además de ostentar esta cualidad, se consideró un corredor natural importante donde ocurre la especie.

Estación Ambiental Tutunendo (Zona I). Se encuentra localizada en el corregimiento de Tutunendo; esta zona pertenece a la subregión fitogeográfica conocida como selva pluvial central, por su ubicación en el borde de la planicie central del Chocó, donde el inicio de la influencia de la cordillera occidental y la fuerte incidencia de los factores climáticos (precipitación) sobre la vegetación, modelan la estructura y la composición del bosque (Gentry 1978).

Geográficamente se sitúa en la parte nororiental del municipio de Quibdó, departamento del Chocó, a 16 km sobre la vía nacional carreteable que de Quibdó conduce a Medellín. Se encuentra ubicada a la margen derecha del río que lleva su mismo nombre, geodésicamente se encuentra a los $05^{\circ} 48^{\prime} 07^{\prime \prime} \mathrm{N}$ y a $\operatorname{los} 76^{\circ} 31^{\prime} 16^{\prime \prime} \mathrm{W}$, presenta una temperatura megatermal, una humedad relativa del $99 \%$ y una precipitación de hasta $11.000 \mathrm{~mm}$ (Rangel 2004) y posee una extensión de $43 \mathrm{~km}^{2}$. Limita por el norte con el río Cabí, por el sur con el río Icho, por el oriente con el corregimiento El 18 y por el occidente con el corregimiento La Troje. Posee una población de 5000 habitantes donde predomina la etnia negra, en menor porcentaje se encuentran indígenas de la etnia Embera y algunos pocos colonos migrantes de otras regiones del país. En toda el área de extensión del municipio y de la EAT, se presentan grandes variaciones en la composición, densidad y distribución de la flora, siendo representativas a nivel de dosel $(20-25 \mathrm{~m}$ de altura) algunas especies maderables como: chanó, trúntrago, carrá, caucho y algarrobo. En area de influencia de la EAT, es común ver remanentes de bosque secundario al lado de cultivos de pancoger, entre algunos relictos de bosque maduro de más de 25 a 30 años donde se hace extracción selectiva de madera.

Estación Ambiental Alto San Juan (Zona II), municipio de Tadó. La Estación Ambiental del Alto San Juan (EAAS), cubre el área de influencia del Consejo Comunitario Mayor de Comunidades Negras de ASOCASAN (municipio de TadóChocó). LaEAAS se sitúa en el corregimiento de Playa de Oro, Tadó, desde donde se extienden las acciones de investigación del IIAP a todo el corredor del Alto San Juan y los municipios aledaños que integran históricamente la subregión del San Juan. El municipio de Tadó se encuentra localizado al nororiente del departamento del Chocó, a los 76³'10" N y a los 5 $16^{\prime} 10^{\prime \prime} \mathrm{O}$ del meridiano de Greenwich, con una altura de $75 \mathrm{msnm}$ y a una distancia de $68 \mathrm{~km}$ de la capital de departamento (Quibdó), presenta una humedad relativa de $84 \%$ aproximadamente, la precipitación oscila entre 4000 mm y 9000 mmal año, la temperatura es variable, entre $27^{\circ} \mathrm{Cy} 32^{\circ} \mathrm{C}$. Tiene una extensión superficial de $1748 \mathrm{~km}^{2}$, corresponde a tierras donde la agricultura se entrecruza con rastrojos, relictos de bosque natural en regeneración y pastizales. Este tipo de asociación mixta, es evidente en el corregimiento de Playa de Oro.

Los habitantes de la localidad implementan cultivos de plátano, banano, piña, caña y borojó. Las asociaciones vegetales que aparecen en esta subregión, están condicionadas por la procedencia del suelo y su posición fisiográfica; en zonas de ladera se desarrolla una asociación con especies de valor comercial. Las especies que caracterizan esta asociación, son: sande (Brosimum utile), carrá (Huberodendron patinoi), sajo (Campnosperma panamensis), palma don pedrito (Oenocarpus minor), palma quitasol (Mauritiella macroclada), tangare (Carapa guianensis), caimitos (Pouteria sp), paco (Cespedesia puthulata), palma naidí (Euterpe oleracea) y chanul (Saccoglotis procera), entre otras. 


\section{Bioetnia Volumen 8 No 1 (enero-junio), 2011}

Corregimiento de Opogodó(Zona III). El corregimiento hace parte del municipio de Condoto. Se encuentra ubicado a $76^{\circ} 39^{\prime} \mathrm{O}$ y $5^{\circ} 03^{\prime} \mathrm{N}$, dista $84 \mathrm{~km}$ del municipio de Quibdó por la carretera que conduce al municipio de Nóvita, sobre la margen izquierda del municipio de Condoto; posee una temperatura promedio de $28^{\circ} \mathrm{C}$, corresponde a un bosque pluvial tropical (bp-T), posee una altitud aproximada de 70 msnm y un régimen de precipitación media anual de $8000 \mathrm{~mm}$ (Arango y Saldarriaga 1997). Limita al norte con el Consejo Comunitario de la Hilaria, al sur con el municipio de Nóvita, al oeste con los Consejos Comunitarios de Guarapito y Jigualito; al oriente con el Consejo de Soledad de Tajuato. El corregimiento de Opogodó, presenta bosques secundarios altamente intervenidos por la minería tradicional y la minería mecanizada. En la parte alta de la cuenca del río Opogodó se pueden encontrar remanentes de bosque primario. La principal actividad económica la constituyen la minería, la actividad agropecuaria, el comercio, turismo y la extracción forestal(EOT municipio de Condoto 2004).

\section{MÉTODOS}

En cada una de las zonas se delimitaron tres transectos lineales que se escogieron al azar en cuadrículas sobre un sistema de información geográfica $(5 \mathrm{~km})$ y tres transectos no lineales escogidos aleatoriamente, basados en la cartografía social de las comunidades (caminos, quebradas, etc.).

Luego se realizaron recorridos y monitoreos constantes. Durante los recorridos diarios se hicieron observaciones directas a la especie para conocer algunos aspectos comportamentales y sociales; en esta actividad se contó con la ayuda de binoculares $(10 \times 40)$ y una mira telescópica. Se determinaron algunos aspectos como comportamiento grupal (hábitos grupales, solitarios, parejas), actividad, hábitat, tipo de dietas, afinidad biogeográfica, estado de conservación y otros aspectos que se registraron sobre un protocolo de campo previamente establecido, además se realizó una caracterización de los hábitats potenciales donde ocurre la especie (bosque secundario, zonas de cultivo y ecotono), así como los diferentes sitios de percha.

\section{RESULTADOSY DISCUSIÓN}

Análisis general del uso del hábitat y aspectos de comportamiento del tucán del Chocó (Ramphastos brevis) en las tres zonas objeto de estudio (selva pluvial tropical del Chocó). Al realizar un análisis general de los hábitats empleados por la especie así como sus conductas de comportamiento en cada una de las localidades evaluadas, se obtuvo como resultado que existen pocas diferencias entre los aspectos objeto de estudio en cada una de las localidades. En su defecto? se identifican los siguientes aspectos para algunas poblaciones del departamento, señalando puntualmente las principales actividades realizadas por el tucán durante el día, así como las diversas especies florísticas y microambientes explorados por la especie en la compleja y biodiversa estructura vegetal chocoana.

Descripción del hábitat de Ramphastos brevis. En las tres localidades estudiadas, se observó que la disponibilidad y la variedad de la oferta trófica al igual que los numerosos árboles propicios para la percha incidieron de forma directa en la observación de los tucanes. Los bosques de las localidades muestreadas se caracterizaron por ser medianamente intervenidos donde predominan las entidades botánicas: Melastomataceas, Piperaceas, Miristicaceas, Musaceas, Arecaceas, y Cecropiaceas; las áreas de bosque donde predominan estas familias están integradas con pequeñas parcelas étnicas donde se siembran variedades de plátano de la familia Musaceae (Musa spp) y algunos frutales entre los que se destacan: piña (Ananas sativa), yuca (Manihots culenta), caña (Chacharum oficinalis), guayaba agria (Psidium araca), borojó (Allibertia patinoi), entre otros. Además de estos frutos objeto de siembra se encontraron forestales de gran importancia comercial como el chano(Humiriastrum procera), el carrá (Huberodendrum patinoi), el lechero (Brosimum sp) y el aserrín (Parkia pendula); la combinación de estos elementos vegetales crea un mosaico vegetal idóneo para el desplazamiento y forrajeo del tucán del Pacífico.

Uso del hábitaty aspectos comportamentales. En las tres zonas de trabajo la especie fue más conspicua en las zonas abiertas, como áreas de cultivo y en el ecotono, la fisionomía del bosque secundario fue una limitante en el avistamiento de la especie. Árboles de gran altura como el aliso (Pollalestea discolor), el yarumo (Cecropia aff peltata), el noanamo (Virolla $\mathrm{sp}$ ) y representantes de la familia Arecaceae, palma mil pesos (Oenocarpus bataua) palma don pedrito (Oenocarpus minor), palma meme (Wettinia quinaria), son los árboles ideales para la percha y la alimentación del tucán; sobre estos árboles se observaron conductas comportamentales de canto, alimentación, forrajeo, limpieza y descanso.

Las zonas de cultivo y el ecotono, parecen poseer una mayor variedad de oferta trófica conforme a los requerimientos básicos de R. brevis; probablemente los cultivos de las localidades estudiadas ofrecen alimento de fácil acceso para el tucán y los cultivos están acompañados por palmas que son fuente de alimento de la especie. Estas palmas también son abundantes en el ecotono, lo que facilita la presencia del ave; desde el punto de vista sistémico, en este lugar se produce el mayor intercambio de energía, lo que favorece la abundancia de la especie, el ecotono representa la zona de máxima interacción entre ecosistemas limítrofes efecto que se refleja en la ocurrencia de la especie. Hilty y Brow 2001 sostienen que $R$. brevis habita en bosques húmedos y a menudo frecuenta 
márgenes o bordes de ecosistemas selváticos.

El tucán visita durante el día diversas especies de palmas propias de la región de las que obtiene sus frutos. En la Estación Ambiental Tutunendo se registró la especie frecuentando a menudo áreas abiertas de cultivo y zonas de bosque secundario, donde son muy abundantes árboles de la familia Cecropiaceae que aprovecha para alimentarse, acicalarse y para descansar. En el corregimiento de Opogodó se encontró que la especie es más frecuente en áreas de poca cobertura vegetal (cultivos, zonas degradas por la minería) y en el eco-tono donde se alimenta sobre todo de un fruto dehiscente conocido vulgarmente como fruta de sábalo o tomé (Dussia macrofilla) y del sangre gallo (Virola cemifera), pertenecientes a la familia Myristicaceae. Allíse encontró que la especie se traga la semilla, que está cubierta por un arilo laciniado de color rojo, lo que le facilita la obtención de la semilla de estos frutos, el tucán solo tiene que presionar el fruto con su fuerte pico.

Este aspecto es similar al encontrado por Hubbell(1979), Schupp (1993), Nathan y Muller-Landau (2000), Bleher y Böhning-Gaese (2001) quienes sostienen que la mayoría de estos animales, por su tipo de dieta, son consumidores de primer grado, porque en valor de importancia los frutos (bayas con semilla grandes y pequeñas, Virola y Cecropia), representan su mayor ítem alimenticio, aportando así a la dispersión de semillas; este aspecto es considerado como uno de los procesos claves que determinan la estructura espacial de las poblaciones de plantas.

$\mathrm{Al}$ igual que en las localidades anteriores, en la Estación Ambiental Alto San Juan se documentó que los tucanes usan o aprovechan para la alimentación algunas entidades botánicas con frutos verdes y maduros, entre las que se encuentran los frutos conocidos como: coronilla (Bellusia sp.), el caucho (Centillia elastica), el sangre gallo (Virola cemifera), palma mil pesos (Oenocarpus bataua), palma don pedrito (Oenocarpus minor), palma meme (Wettinia quinaria) y fruto de sábalo (Dussia macrofilla); todos estos frutos son parte de la oferta y la demanda trófica de la especie y asimismo, los árboles que los contienen se emplean previamente en actividades de comportamiento. También se registraron sobre estos árboles maniobras de agilidad donde el ave mueve fuertemante el pico a la hora de alimentarse, algo similar a lo encontrado por Hubbell (1979), Schupp (1993), Nathan y Muller-Landau (2000), Bleher y Böhning-Gaese (2001), quienes sostienen que la especie forrajea todos los niveles del bosque y es muy ágil cuando se alimenta usando su largo pico que le confiere una buena ventaja cuando está buscando frutos (Ridgely y Greenfield 2001).

Sin embargo, y a pesar de esta clase de aspectos encontrados, no se lograron obtener registros de caza o consumo de presas o animales vivos en ninguna de las zonas de trabajo, como sí se registró en un estudio de Ridgely y Greenfield
(2001), quienes sostienen que estos tucanes pueden también comportarse como consumidores de segundo grado, porque complementan su dieta con invertebrados (hormigas y otros insectos), vertebrados (huevos de vertebrados y polluelos de otras especies de aves que encuentran en otros nidos) (Remsen et al. 1993, Van Dorp 1985). Este importantísimo aspecto, aunque no se registró en campo, fue confirmado por expertos cazadores de la región que sostienen que Rhamphastos brevis y otras especies de tucanes suelen saquear los nidos de muchas especies de aves entre las que distinguen el mochilero (Cacicus cela) y el titiribí (Tyranus melancholicus).

Anotaciones, características del hábitat y sitios de percha. La distribución o segregación de árboles o entidades botánicas usadas como perchas por R. brevis, es aleatoria o secuencial y se presenta acorde con los procesos de segregación dados en cada uno, proceso en el que interviene el túcan al contribuir en la de dispersión de estas semillas.

Por otra parte, las actividades antrópicas desarrolladas en los hábitats de las localidades estudiadas, tienen alta incidencia en la continuidad del paisaje natural y no permiten distinguir una cualidad general del bosque en particular, puesto que actividades como la extracción selectiva de árboles maderables, la minería artesanal y mecanizada, la expansión agrícola y en baja proporción la ganadería, no tienen ningún tipo de planificación o delimitación espacial, y se desarrollan de acuerdo con las condiciones de vida, requerimientos y necesidades de las comunidades; este evento no permite distinguir extensas unidades homogéneas de paisaje, sino que más bien se observa un mosaico de bosque natural, en medio de áreas cultivadas y degradadas por las actividades mencionadas.

Conforme a lo anterior, es pertinente anotar que el túcan del Chocó, ocupa todos los escenarios tratados, pues en cada uno de estos se encontraron los árboles que requiere para sus actividades diarias; paradójicamente esta especie endémica de bosque húmedo tropical, es más conspicua en sitios abiertos donde es más fácil encontrarla. Por lo general, cuando se observó en lugares cerrados o boscosos, fue a través de casos fortuitos y en estos escenarios se registró que son bastante quietos y silenciosos.

El tucán del Chocó $R$. brevis se observó principalmente en grupos de dos a seis individuos (raramente solitarios). En múltiples ocasiones se registró forrajeando acompañado de otros Ramphastidos: Ramphastos swainsonii (diostedé), Pteroglossus sanguineus (pichi), alimentándose de frutos a alturas entre los 10-30 m; en una ocasión en la Estación Ambiental Tutunendo, se registraron volando a $5 \mathrm{~m}$ de altura. Según Ridgely y Greenfield (2001) la especie forrajea todos los niveles del bosque, es muy ágil cuando se alimenta usando su largo pico que le confiere una buena ventaja cuando está buscando frutos. Los individuos observados nunca produjeron llamados de alarma u otro tipo de vocalización, solo se 


\section{Bioetnia Volumen 8 No 1 (enero-junio), 2011}

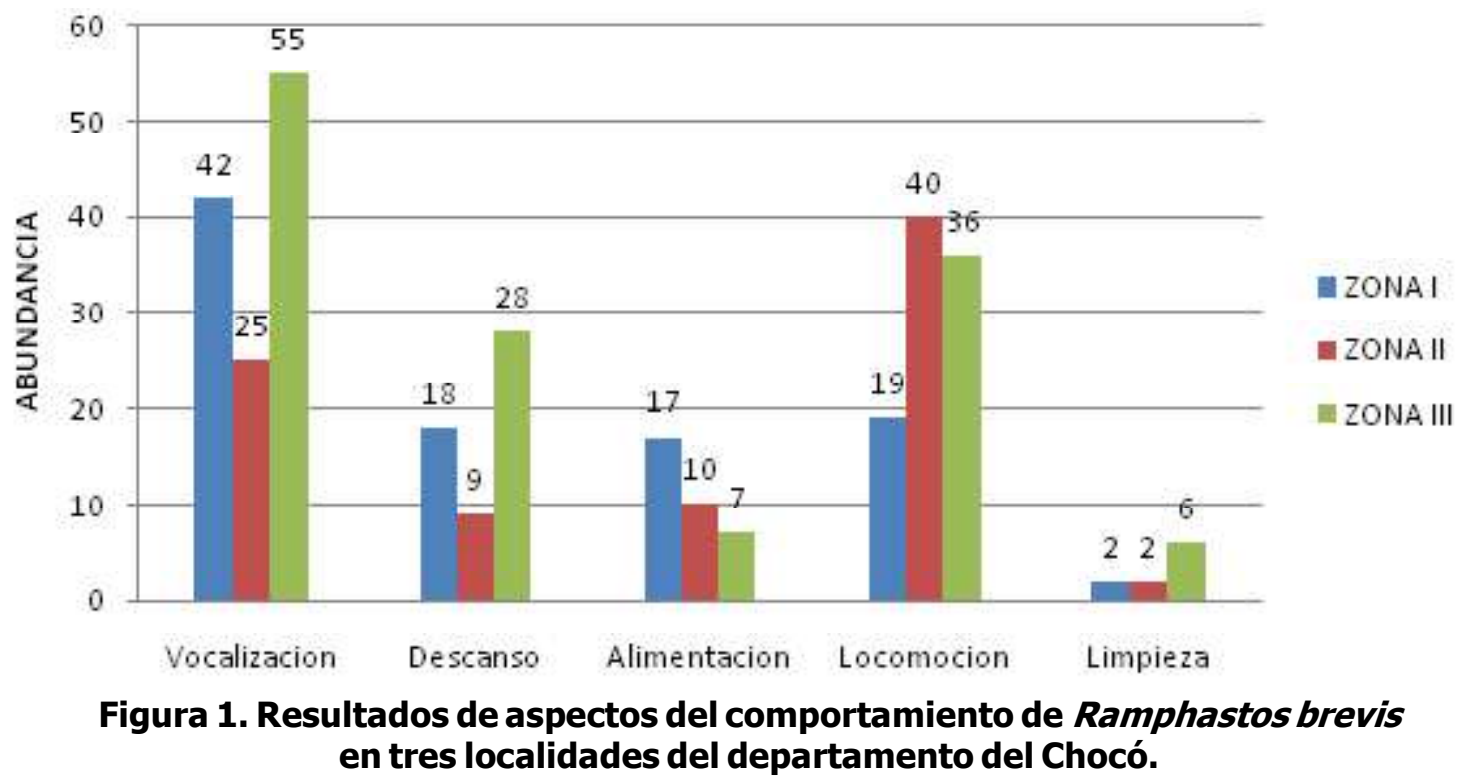

movieron silenciosamente a través de la vegetación. En la EAT se colectaron muestras fecales húmedas, junto con otras secas, las muestras contenían semillas de Cecropiaceae (yarumo), lo que hace pensar que la especie dentro de sus actividades de forrajeo presenta fidelidad de percha en ciertos árboles (porque las muestras fecales se obtuvieron al mismo tiempo y su aspecto hace pensar que no fueron excretadas el mismo día). Se pudo observar que la dieta de $R$. brevis está compuesta por frutos, destacándose los de la especie Cecropia aff. Peltata, que es muy abundante en la zona.

Dentro del análisis comportamental de la especie también se registró que la vocalización fue la conducta etológica más observada en las zonas I y III, mientras que en la zona II fueron más perceptibles los tucanes por el movimiento. El segundo lugar en acciones de comportamiento lo ocupó este mismo aspecto (la locomoción), es decir, que tanto la vocalización como la locomoción fueron las actividades de mayor observación en $R$. brevis (Figura 1). Los despliegues acústicos de estos individuos se registraron durante todo el día con picos máximos de actividad en las horas de la mañana entre las 7:00 am y las 12:00 m, aunque estos llamados suelen presentarse durante todo el día hasta aproximadamente las 5:50 pm. Durante los registros se encontró que las vocalizaciones que emiten dependen en buena medida de variables atmosféricas como la precipitación y el brillo solar excesivo, períodos en que las vocalizaciones suelen disminuir considerablemente. Los pobladores de la localidades estudiadas sostienen que en ausencia de lluvia estas aves vocalizan para que la lluvia caiga.

Otras conductas de comportamiento como descanso, alimentación y limpieza respectivamente, se observaron en menor proporción y tienen estrecha relación con la vocaliza- ción, puesto que en reiteradas ocasiones, después de haber observado los individuos (por vocalización) estos se registraron forrajeando en busca de alimento o descansando bajo la sombra de palmas o Cecropias spp.

Durante el monitoreo se observó que el horario de mayor actividad del tucán del Pacífico es entre las 7:00 am y las 10:00 am. Durante este período son mucho más visibles y aprovechan fuentes de alimentos que se encuentran en las pequeñas parcelas y fincas cercanas a los asentamientos humanos; a medida que avanza la mañana hay disminución en la observación, tienden a ocultarse en zonas más boscosas donde esta es mucho más difícil y por esa razón se nota un decrecimiento en la gráfica a medida que transcurre el día (Figura 2).

Durante este estudio se evidenció la importancia de trabajos de comportamiento, que permiten la reconstrucción de aspectos relevantes de la historia natural de la especie. Otros elementos comportamentales como las estrategias reproductivas de las que no se tuvo evidencia, constituyen vacíos de información que se deben documentar en futuras investigaciones.

\section{CONCLUSIONES}

Los hábitats de mayor uso del tucán del Chocó Rhamphastos brevis, son el ecotono y zonas abiertas de cultivo donde la especie aprovecha las primeras horas del día para forrajear y alimentarse; en contraste, la fisionomía vegetal del bosque secundario es una limitante en el avistaje de la especie.

En los hábitats de mayor ocurrencia el tucán, aprovecha los frutos de algunas especies vegetales de la familia Arecaceae y de la familia Cecropiaceae, y de paso utiliza estos árboles 


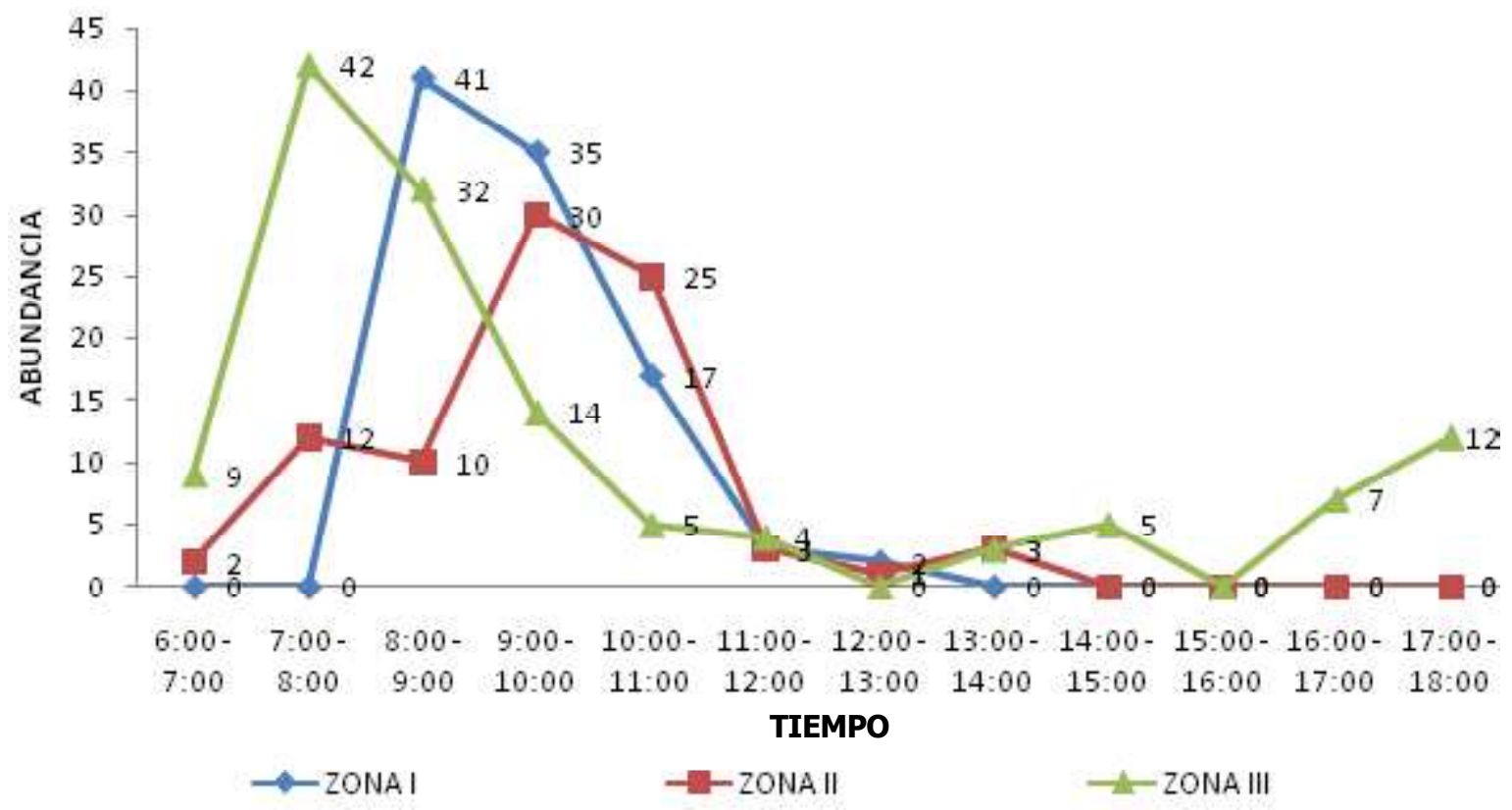

\section{Figura 2. Picos de actividad de Ramphastos brevisen tres localidades del departamento del Chocó.}

para realizar actividades de descanso, vocalización, limpieza y acicalamiento.

El tucán del Chocó es un ave social que forrajea los estratos más altos del bosque donde forrajea activamente con varios coespecíficos y en múltiples ocasiones se le puede ver con otros Ramphastidae como el diostede (R. swaisonii) y el pichi (Pteroglosus sanguineus).

Las actividades de mayor observación en la especie fueron la vocalización y la locomoción que realiza en las primeras horas del día. A medida que avanza el día estas actividades decrecen, siendo entre las 7:00 am y las 10:00 am, el período de mayor actividad de forrajeo de la especie.

El corto tiempo del estudio fue quizás una limitante en la observación de otras actividades de comportamiento de la especie como por ejemplo la reproducción; sin embargo, otras actividades encontradas en la literatura, como el saqueo de huevos y nidos de otras aves, fueron confirmadas por expertos cazadores de las localidades, que sostienen que las aves más afectadas por este tipo de conducta son el mochilero (Cacicus cela) y el titiribí (Tyranus melancholicus).

\section{AGRADECIMIENTOS}

Se agradece a los Consejos Comunitarios locales de Tutunendo, área de influencia de la Estación Ambiental de
Tutunendo, ASOCASAN, área de influencia de la Estación Ambiental de Alto San Juan y Consejo Comunitario de Opogodó, al igual que a la Agencia Nacional de Hidrocarburos, con su empresa perforadora GPC, Drilling y el Instituto de Investigaciones Ambientales del Pacífico.

\section{LITERATURA CITADA}

Arango, D., T. Saldarriaga. 1997. Monografía del Chocó. Quibdó: Editorial de Autores Chocoanos. 78 pp.

EOTC. 2004. Esquema de Ordenamiento Territorial Condoto. Quibdó: Instituto de Investigaciones Ambientales del Pacífico. 189 pp.

Hilty, S. L., W. L. Brown. 2001. Guía de las aves de Colombia. Princeton University Press, American Bird Conservancy-ABC, Universidad del Valle, Sociedad Antioqueña de Ornitología (SAO).

Hubbell, S. 1979. Tree dispersión, abundance and diversity in a tropical dry forest. Science 203: 1299-309.

McMullan, M., T. M. Donegan, A. Quevedo. 2010. Field guide to the birds of Colombia. Bogotá: Fundación ProAves de Colombia. $82 \mathrm{pp}$.

Rangel, J. O. (Ed). 2004. Colombia Diversidad Biótica, IV. El Chocó biogeográfico/costa Pacífica. Bogotá: Instituto de Ciencias Naturales de la Universidad Nacional de Colombia, Conservación Internacional. 997 pp.

Restall, R., C. Rodner, M. Lentino. 2006. Birds of northern South America: An identification guide. Vol. 2. London: A\&C Black Helm. 25 pp.

Ridgely, R., J. Jr. Gwynne. 1993 (edición en español). Una guía de las aves de Panamá incluyendo Costa Rica, Nicaragua y Honduras. ANCON. En línea [fecha de acceso: 21 de noviembre de 2010]. URL disponible en: http:/ /biota.wordpress.com/2007/10/06/los-tucanes-de-panama-familiaramphastidae/ 